\title{
Experimental and parametric study of a solar paraboloid designed to receive a Stirling engine
}

\author{
Djelloul Azzouzi ${ }^{1, a}$, Boussad Boumeddane ${ }^{1}$, Abderahmane Abene ${ }^{2}$ \\ AND NouredDine SAID $^{3}$ \\ 1 Faculty of Engineering Sciences, University of Blida, Algeria \\ 2 Valenciennes Institute of Sciences and Techniques, University of Valenciennes, France \\ ${ }^{3}$ Centre of Renewable Energies Development, Algiers, Algeria
}

Received 13 October 2014, Accepted 22 October 2014

\begin{abstract}
An investigation for the parametric characterization of a solar paraboloid intended to receive a Stirling engine was studied in this work. This one comprises two principal parts; the first one consists of the design of a solar parabolic concentrator having an aperture area of $1.67 \mathrm{~m}^{2}$ with a focal distance of $52.65 \mathrm{~cm}$. Its primary surface was covered by 152 aluminium facets distributed uniformly on eight equal petals. The optical parameters revealed a peak concentration ratio of 40488 , while average concentration ratio is 4920 . The real focal distance and the dimension of the sunspot focused at the focal zone are given in experiments after a series of tests. The second part was devoted to the development of an analytical model based on the resolution of the heat equation using the variables separation method. This model adopted is able to predict the distribution of temperature and heat flux at the focal zone. By the application of Soltrace code, it is noted that the predicted results by the analytical model are in good agreement with those obtained by experiments.
\end{abstract}

Key words: Parabolic concentrator / receiver flat / radiative flux / ray tracing

\section{Introduction}

The solar parabolic concentrators present better optical and thermal efficiencies compared to other solar concentrators [1]. This is primarily due to their mode of specific concentration which supports the rational collection of the incidental rays in the focal zone compared with the mode of linear concentration [2]. When designing a parabolic concentrator, it is very significant to use sizes of facets which can ensure a coherent distribution within the primary surface of the concentrator in order to generate the highest concentration ratio [3], and to reduce the useless surface [4] generated by the inter facets junctions. The optical and thermal characterization of the parabolic concentrators is made using several experimental and numerical methods able to determine the real focal distance and to predict the flux distribution at the focal zone. One of these experimental methods consists in measuring average heat flux using the charge coupled device imaging cameras [5]. The ray tracing technique based on the method of Monte Carlo is largely used, it became one of the powerful tools used for characterization and optimizations of various parameters [6-8] intervening in the solar concentration and it makes possible to model

\footnotetext{
a Corresponding author: azzouzidjelloul@yahoo.fr
}

a broad range of concentrators. In this work we present the design stages of the solar parabolic concentrator followed by an experimental investigation to determine the real focal distance and the heat flux intensity focused at the focal zone. A numerical model based on an analytical development was developed with the order to predict the temperature distribution and heat flux at the focal zone. The Soltrace code using ray tracing technique was employed in the determination of the peak heat flux and flux distribution at the receiver flat, making it possible to compare the results obtained by experiments with those predicted numerically.

\section{Materials and methods}

\subsection{Solar concentrator design}

The solar concentrator was designed in collaboration with the Centre of Renewable Energies Development. Its aperture diameter is $146 \mathrm{~cm}$ and a depth of $25.3 \mathrm{~cm}$. The realization of this solar concentrator proceeded while passing by the following construction steps:

- polishing of the primary surface of the parabolic concentrator; 


\section{Nomenclature}

\begin{tabular}{|c|c|c|c|}
\hline$\overline{A_{\mathrm{p}}}$ & Paraboloid primary surface $\left(\mathrm{m}^{2}\right)$ & $\dot{Q}_{\mathrm{ra}}$ & Radiative power (W) \\
\hline$A_{0}$ & Concentrator aperture area $\left(\mathrm{m}^{2}\right)$ & $\dot{q}$ & Heat energy $(\mathrm{W})$ \\
\hline$A_{\mathrm{s}}$ & Sunspot area $\left(\mathrm{m}^{2}\right)$ & $\dot{q}_{\text {avg }}$ & Average heat flux $\left(\mathrm{W} \cdot \mathrm{m}^{-2}\right)$ \\
\hline$a$ & Thermal diffusivity $\left(\mathrm{m}^{2} . \mathrm{s}^{-1}\right)$ & $\dot{q}_{\mathrm{s}}$ & Heat flux $\left(\mathrm{W} \cdot \mathrm{m}^{-2}\right)$ \\
\hline$C$ & Concentration ratio & $T$ & Temperature $(K)$ \\
\hline$C_{\max }$ & Maximal concentration ratio & $T_{\max }$ & Maximal temperature $(\mathrm{K})$ \\
\hline$C_{\text {mean }}$ & Mean concentration ratio & $T_{r}$ & Receiver temperature $(\mathrm{K})$ \\
\hline$\left(C_{\mathrm{p}}\right)_{\mathrm{r}}$ & Specific heat of the receiver flat $\left(\mathrm{J} . \mathrm{Kg}^{-1} \cdot \mathrm{K}^{-1}\right)$ & $T_{\infty}$ & Ambient temperature $(\mathrm{K})$ \\
\hline$\left(C_{\mathrm{p}}\right)_{\text {air }}$ & Specific heat of air $\left(\mathrm{J} \mathrm{Kg}^{-1} \cdot \mathrm{K}^{-1}\right)$ & $t$ & Time $(s)$ \\
\hline$d$ & Diameter aperture of the concentrator $(\mathrm{m})$ & $\Delta V$ & Elementary volume $\left(\mathrm{m}^{3}\right)$ \\
\hline \multirow[t]{2}{*}{$d_{\mathrm{s}}$} & Diameter of sunspot (m) & $x, y$ & Spatial coordinates $(\mathrm{m})$ \\
\hline & & \multicolumn{2}{|r|}{ Greek symbols } \\
\hline$E_{\mathrm{s}}$ & Incident direct solar flux $\left(\mathrm{W} . \mathrm{m}^{-1}\right)$ & $\alpha_{\text {eff }}$ & Effective absorptance \\
\hline$e$ & Thickness of the receiver flat $(\mathrm{m})$ & $\beta$ & Coefficient of thermal expansion $\left(\mathrm{K}^{-1}\right)$ \\
\hline$f$ & Focal length (m) & $\varepsilon_{\mathrm{r}}$ & Emissivity of the receiver surface \\
\hline$G r$ & Grashof number & $\theta$ & Angle of the solar disc (rad) \\
\hline$g$ & Acceleration of gravity $\left(\mathrm{m} . \mathrm{s}^{-1}\right)$ & $\mu$ & Dynamic viscosity $\left(\mathrm{kg} \cdot \mathrm{m}^{-1} \cdot \mathrm{s}^{-1}\right)$ \\
\hline$h$ & Convective heat transfer coefficient $\left(\mathrm{W} \cdot \mathrm{m}^{-2} \cdot \mathrm{K}^{-1}\right)$ & $v$ & Kinematic viscosity $\left(\mathrm{m}^{2} \cdot \mathrm{s}^{-1}\right)$ \\
\hline$K$ & Thermal conductivity $\left(\mathrm{W} \cdot \mathrm{m}^{-1} \cdot \mathrm{K}^{-1}\right)$ & $\rho$ & Density $\left(\mathrm{Kg} \cdot \mathrm{m}^{-3}\right)$ \\
\hline$L$ & Length of the receiver flat $(\mathrm{m})$ & $\bar{\rho}$ & Reflectance of the concentrator \\
\hline$N u$ & Nusselt number & $\sigma$ & Stefan-Boltzman constant $\left(\mathrm{W} \cdot \mathrm{m}^{-2} \cdot \mathrm{K}^{-4}\right)$ \\
\hline$L$ & Length of the receiver flat $(\mathrm{m})$ & $\theta$ & Angle of the solar disc (rad) \\
\hline $\operatorname{Pr}$ & Prandtl number & $\psi$ & Rim angle of paraboloid (rad) \\
\hline
\end{tabular}

- division of primary surface in eight equal petals;

- whole division of the aluminium facets in six types distributed uniformly on the eight petals of the concentrator;

- joining the facets with a good adherence on the polished primary surface of the concentrator, by respecting the position of each facet on this surface.

The size of each facet type and its position are carefully selected in order to ensure a good adherence with the primary surface of the parabolic concentrator during joining stage as indicated in Figure 1 which shows the six various aluminium facets.

This setting stage of the facets on polished primary surface has a great role in the optical effectiveness of the concentrator, because any imperfection generates the dispersion of the solar rays reflected through the reflective surface of the concentrator, and consequently a considerable reduction in flux concentrated at the focal zone. In the same way, it should be noted that the azimuth and radial position of each facet are respected in order to reduce the useless surface [4] generated by the inter facets junctions.

\subsection{Geometrical parameters}

The reflecting surface of the concentrator as indicated in Figure 2 is covered with 152 facets of aluminium reflectors distributed into six various rows. The thickness of each facet is $0.8 \mathrm{~mm}$ while the aperture area is $1.67 \mathrm{~m}^{2}$. The addition of the different surface facets gives a total reflective concentrator surface of $1.77 \mathrm{~m}^{2}$. Starting from the third row it is to be noticed that, more we move away from the basic center of the paraboloid, more the facets size increases in order to ensure a good adherence of each facet with the primary surface of the parabolic concentrator. Moreover, if the number of facets increases for the same primary surface of the concentrator, the reflective surface decreases appreciably [3], this is due to the number of existing gaps between the facets. This report can be checked by a simple difference between the uncovered primary surface of the paraboloid calculated by equation (1), and its reflective surface which appears in Table 1 . This difference of $830 \mathrm{~cm}^{2}$ corresponds to a reduction of about $4 \%$ of the primary surface

$$
A_{\mathrm{p}}=\frac{8}{3} \pi f^{2}\left[\left(\left(\frac{D}{4 f}\right)^{2}+1\right)^{3 / 2}-1\right]
$$

where $f$ is the focal length and $D$ is the aperture diameter as indicated in Figure 2.

The facets number which each one of the six rows contains and their corresponding surfaces are illustrated in Table 1.

\subsection{Optical parameters}

The dominant optical parameter in the solar concentrator is the solar concentration ratio which is defined as the solar flux at the focal plan divided by the direct normal solar irradiation. According to Bliss [9] the coefficient of solar concentration $C$ reaches its maximum value for 


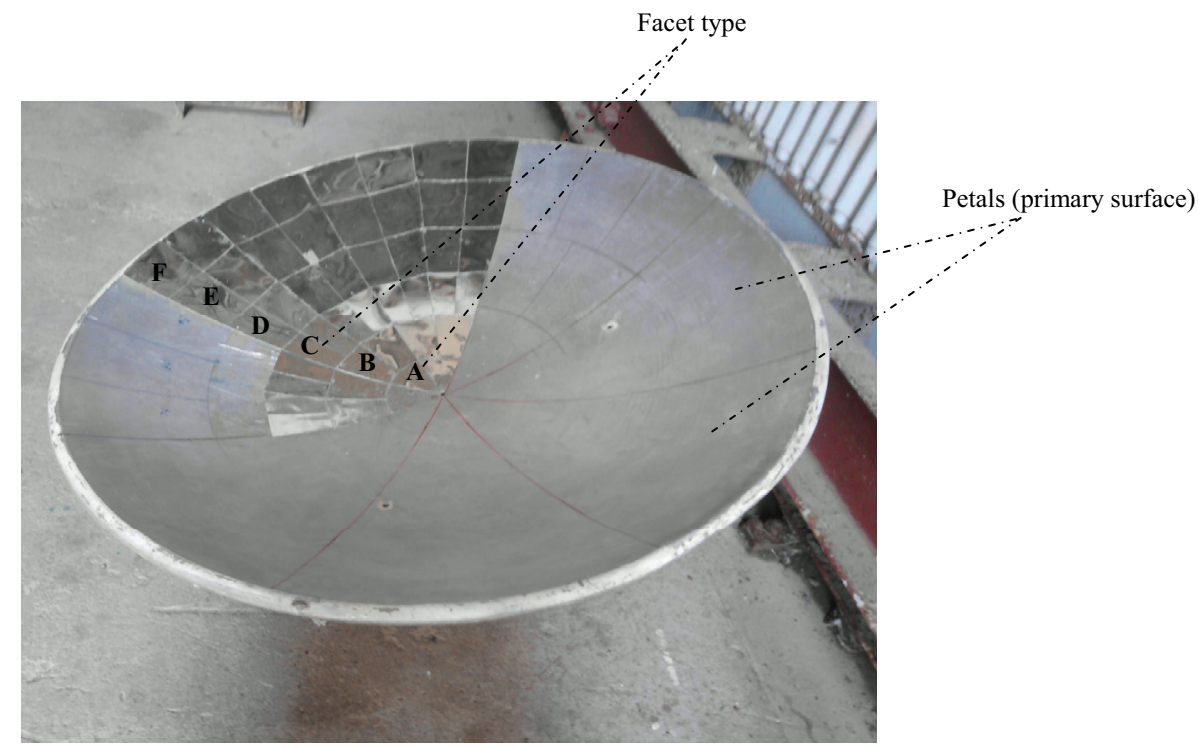

Fig. 1. Image of he concentrator under realization.

Table 1. Number and surface of each facet type of the reflective concentrator surface.

\begin{tabular}{cccc}
\hline Facet type & Facets number & Facet surface $\left(\mathrm{cm}^{2}\right)$ & Total surface $\left(\mathrm{cm}^{2}\right)$ \\
\hline A & 8 & 50.52 & 404.16 \\
B & 16 & 88.39 & 1414.24 \\
C & 32 & 80.36 & 2571.52 \\
D & 32 & 107.55 & 3441.60 \\
E & 32 & 147.72 & 4727.04 \\
F & 32 & 163.01 & 5216.32 \\
\hline \multicolumn{2}{l}{ Total reflective surface of concentrator $\left(\mathrm{cm}^{2}\right)$} & 17774.88 \\
\hline
\end{tabular}

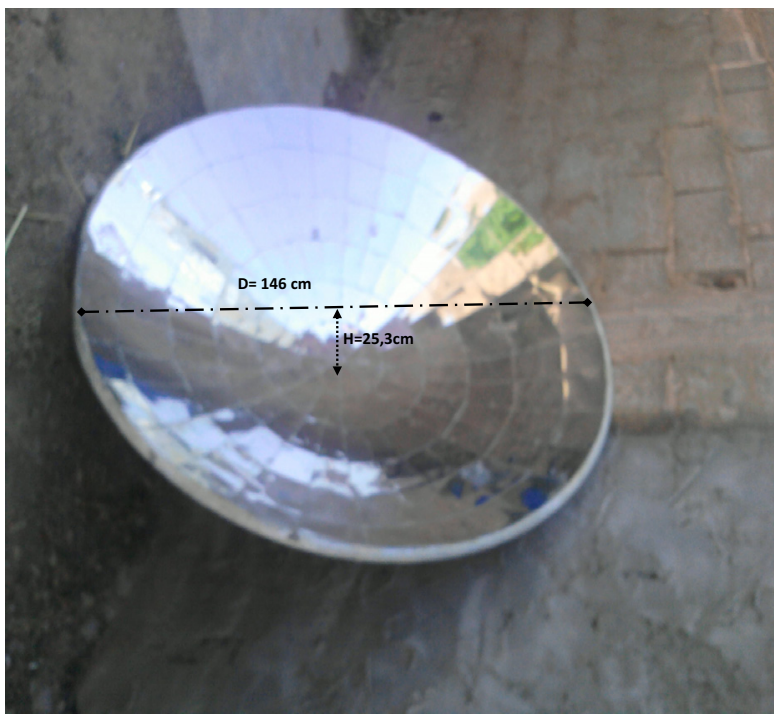

Fig. 2. Image of the designed solar paraboloid.

an ideal solar paraboloid focusing solar flux in the form of circular image (spot) at the focal zone. The theoretical diameter of the spot is given by the following formula:

$$
d_{\mathrm{s}}=f \theta
$$

where $\theta$ is the angle of the solar disc equal to 0.00931 radian.

The peak solar ratio is expressed by the analytical relation developed by Bliss [9]:

$$
C_{\max }=\frac{4}{\theta^{2}} \sin ^{2}(\psi)
$$

while the mean concentration ratio on the receiver surface which takes into account the uniform heat flux distribution in all formed sunspot [4], is given by the analytical expression:

$$
C_{\text {mean }}=\frac{\sin ^{2}(\psi) \cos ^{2}\left(\psi+\frac{\theta}{2}\right)}{\sin ^{2}\left(\frac{\theta}{2}\right)}
$$

The maximum value of $C_{\text {mean }}$ is reached for a rim angle of about $45^{\circ}$ [10] for an ideal paraboloid with flat receiver. For receivers with other geometries [11] the optimal rim angle is not necessarily at $45^{\circ}$. By applying equation (4), the mean concentration ratio relating to our parabolic concentrator is about 4920 . This solar concentration ratio is affected by several parameters $[3,12,13]$ in which some of them are cited below:

- the reflectivity of the aluminized surface of the parabolic concentrator; 


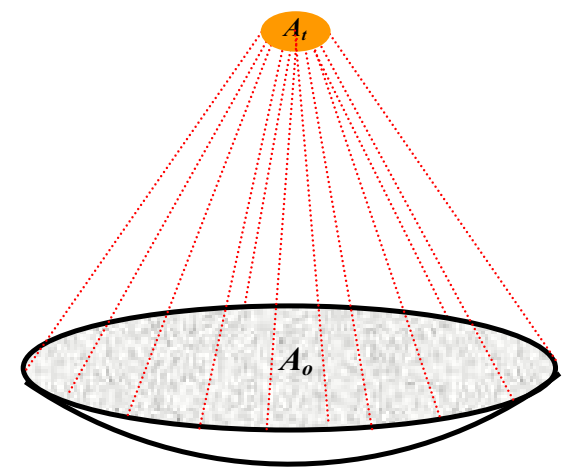

Fig. 3. Scheme of a focal concentration.

Table 2. Geometrical and optical parameters of the solar.

\begin{tabular}{lr}
\hline Aperture area & $1.67 \mathrm{~m}^{2}$ \\
Reflecting surface & $1.77 \mathrm{~m}^{2}$ \\
Focal length & $52.65 \mathrm{~cm}$ \\
Rim angle & 69.5 \\
Number of facets & 152 \\
Number of rows & 6 \\
Concentrator reflectivity & 0.94 \\
Average concentration ratio & 4920 \\
\hline
\end{tabular}

- the facets number and the slope errors;

- the imperfection due to the geometry of the aluminium facets;

- the concentrator positioning errors.

The knowledge of the solar concentration ratio makes it possible to determine the theoretical surface of the focused sunspot [14], by the application of equation (5) which expresses the solar concentration ratio as the report between the aperture area $A_{0}$ and sunspot surface $A_{t}$ as illustrated in Figure 3

$$
C=\frac{A_{0}}{A_{\mathrm{s}}}
$$

In Table 2 are illustrated the geometrical and optical parameters of the designed parabolic concentrator. The reflectivity is provided by the reflector manufacturer.

\subsection{Thermal parameters}

The important thermal parameters in the characterization of a solar concentrator are the solar flux intensity and the temperature field at the focal zone. They are appreciably affected by the value of the direct normal insolation (DNI), the concentrator orientation as well as the solar concentration ratio. The theoretical evaluation of the maximum temperature is based on the following assumptions:

1. the incidental solar power $\dot{Q}_{\text {in }}$ of the paraboloid is completely received by the sunspot surface $A_{t}$;

2 . the cavity receiver of an aperture area $A_{t}$ positioned in the focal plan is thermically insulated in order to eliminate any heat loss by convection or conduction;

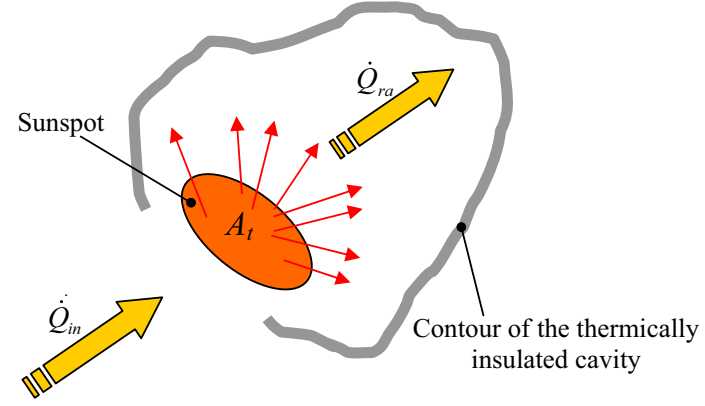

Fig. 4. Descriptive scheme of a cavity receiver perfectly insulated.

3. the temperature distribution is considered homogeneous in all surface of sunspot.

In the absence of any heat transfer by convection and conduction in the insulated cavity, the incidental solar power $\dot{Q}_{\text {in }}$ (Fig. 4) is completely converted into radiative heat $\dot{Q}_{\text {ra }}$ generated by radiation of the sunspot surface. The heat balance of the cavity is expressed by the following equation:

$$
\dot{Q}_{\text {in }}=\alpha_{\text {eff }} \dot{Q}_{\text {ra }}
$$

where $\alpha_{\text {eff }}$ is the effective absorptance of the cavity receiver [15], defined as the fraction of energy entering through the aperture that is absorbed by the cavity walls.

We have:

$$
\bar{\rho} E_{\mathrm{s}} A_{0}=\alpha_{\mathrm{eff}} \varepsilon \sigma A_{\mathrm{s}} T^{4}
$$

where $\bar{\rho}$ and $\varepsilon$ are respectively the reflectivity of the concentrator and the emittance of the cavity receiver taken as a black body, $T$ is the nominal receiver temperature, and $\sigma$ is the Stephan-Boltzmann constant which is equal to $5.67 \times 10^{-8} \mathrm{~W} \cdot \mathrm{m}^{-2} \cdot \mathrm{K}^{-4}$. When we introduce equation (5) into equation (7) and takes $\alpha_{\text {eff }}$ equal to 1 , it results that the maximum temperature reached is expressed by:

$$
T_{\max }=\left(\frac{\bar{\rho} E_{s}}{\varepsilon \sigma} C\right)^{\frac{1}{4}}
$$

Figure 5 represents the variation of the cavity temperature with the solar concentration ratio $C$. It is noted that when $C$ varies, the temperature varies differently in two distinct zones. The first zone (I) is characterized by a faster rise in the temperature at the focal point. In the interval from 50 to 1200 of the solar concentrations ratio, we record a rise of temperature from $1000 \mathrm{~K}$ to $2120 \mathrm{~K}$ that is a rise rate around $0.97 \mathrm{~K}$ by concentration ratio unit. The second zone (II) is characterized by a slowed down development of the temperature in a range of concentration between 1200 and 5800. We record a rise rate about $0.29 \mathrm{~K}$ by concentration ratio unit, while for concentrations higher than 6000 , the rise rate is increasingly low. The application of equation (8) stipulates that the temperature of the neighbouring solar disc of $5800 \mathrm{~K}$ [15] is theoretically reachable by means of a parabolic concentrator able to develop a solar concentration ratio about 67400 . However this temperature cannot 


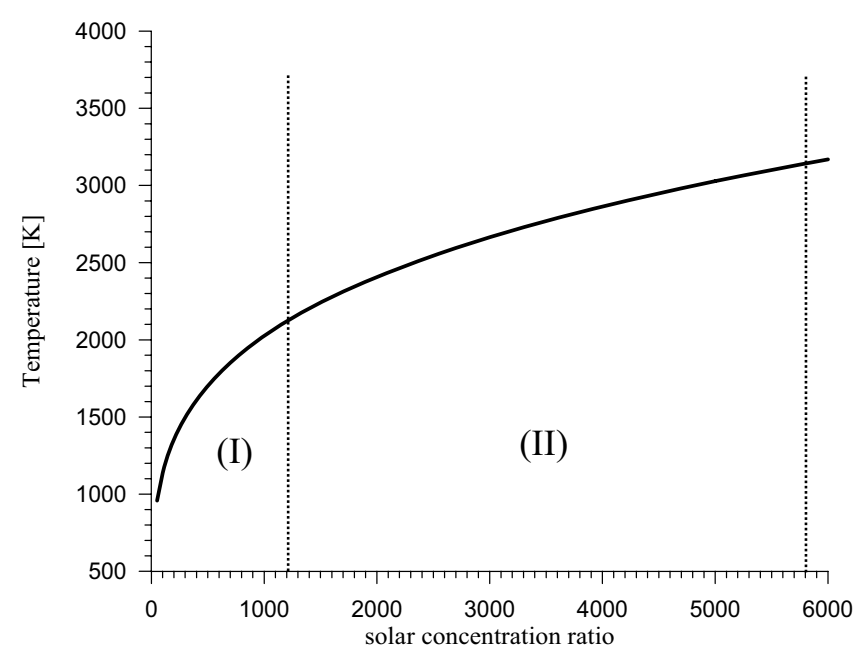

Fig. 5. Maximum temperature at the focal point according to solar concentration ratio.

be reached because the maximum concentration ratio that an ideal parabolic concentrator can develop, according to Bliss [9] can hardly exceed 46148 by the application of equation (3).

\section{Experimental analysis}

The designed solar paraboloid represented in Figure 2 was the subject of an experimental characterization in order to determine its optical and thermal parameters. The first of its parameters is the focal distance which is defined by the length which connects the basic center of the paraboloid to the sunspot center as indicated in Figure 6. For its determination, we placed a positioning mechanism along the focal axis. At the upper part a mild steel metal flat of rectangular form is placed on which concentrates the incidental solar flux. In Table 3 appear the dimensions and the thermophysical properties of the receiver flat. At the center of the receiver flat which has a thickness of $1.8 \mathrm{~mm}$ is placed a thermocouple of type $\mathrm{K}$ $\left(-50^{\circ} \mathrm{C}\right.$ to $\left.1200^{\circ} \mathrm{C}\right)$. This latter is used to measure the focal point temperature at various distances started from $41 \mathrm{~cm}$ to $55 \mathrm{~cm}$, with a variable step between $3 \mathrm{~cm}$ and $0.75 \mathrm{~cm}$. The experimental tests were carried out under a direct normal insolation of $956 \mathrm{~W} \cdot \mathrm{m}^{-2}$ measured by the CMP11pyranometre. A series of tests was started during this day, by taking the temperature at each distance.

It should be noted that more we approach in the focal plan more we reduce the step of measurement in order to determine the focal length with a better possible precision.

The positioning and the orientation of the parabolic concentrator are carried out with a solar tracking system, while the maximum temperature is reached when the geometrical center of the receiver flat coincides exactly with the focal point.

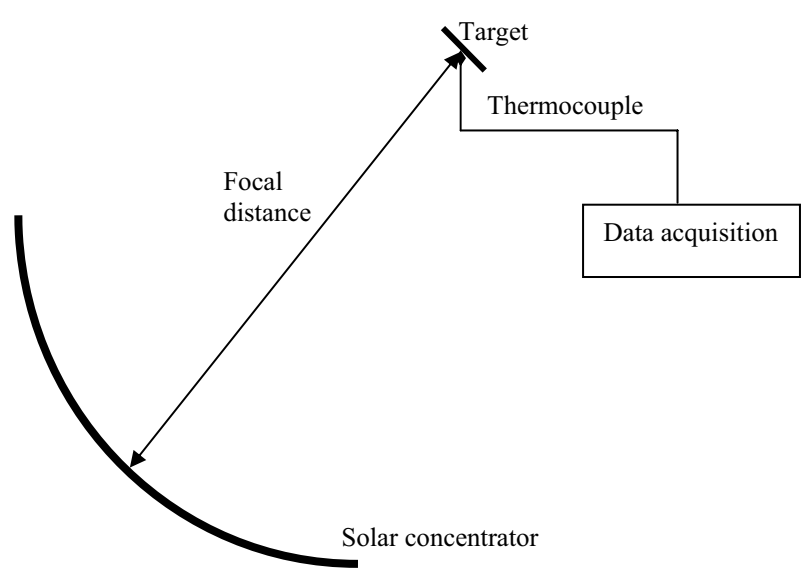

Fig. 6. Schematic of the temperature measurement at the focal point.

Table 3. Specifications of the receiver flat.

\begin{tabular}{lr}
\hline Size & $10 \times 10 \mathrm{~cm}$ \\
Thickness & $1.8 \mathrm{~mm}$ \\
Density & $7850 \mathrm{Kg} \cdot \mathrm{m}^{-3}$ \\
Thermal conductivity & $1.15 \mathrm{~W} \cdot \mathrm{m}^{-1} \cdot{ }^{\circ} \mathrm{C}^{-1}$ \\
Specific heat & $0.458 \mathrm{KJ} . \mathrm{kg}^{-1} \cdot{ }^{\circ} \mathrm{C}^{-1}$ \\
Effective absorptance & 0.92 \\
\hline
\end{tabular}

\section{Analytical development}

The temperature field at the focal zone is of great importance, as it allows a suitable determination of the aperture area size [16] of the cavity receiver which is largely used as a hot source in the solar Stirling engines. This temperature distribution in a receiver flat positioned at the focal zone, and exposed to a constant heat flux, is obtained by the resolution of the equation of unsteady heat transfer expressed in the following form:

$$
\frac{\partial^{2} T}{\partial x^{2}}+\frac{\partial^{2} T}{\partial y^{2}}=\frac{1}{a} \frac{\partial T}{\partial t}
$$

The receiver flat of square form having a thickness $e$ and a length $L$ supposed as an infinite solid with the ratio $\frac{e}{L} \ll 1$. At time $t=0$, the temperature field is characterized by $T(x, y)=T_{0}$, while the boundary conditions are of Dirichlet type as indicated in Figure 7

$$
\begin{array}{llll}
x=0 & \text { and } & 0 \leqslant y \leqslant L & T(0, y)=T_{\infty} \\
y=0 & \text { and } & 0 \leqslant x \leqslant L & T(x, 0)=T_{\infty} \\
x=L & \text { and } & 0 \leqslant y \leqslant L & T(L, y)=T_{\infty} \\
y=L & \text { and } & 0 \leqslant x \leqslant L & T(x, L)=T_{\infty}
\end{array}
$$

By applying the separation variables method, we can find the solution of the heat equation in the form of product of two independent functions given by the following expression:

$$
T(x, y, t)=F(x, y) G(t)
$$

After derivation and replacement in equation (9), passing by a succession of integration and changes of variable, 


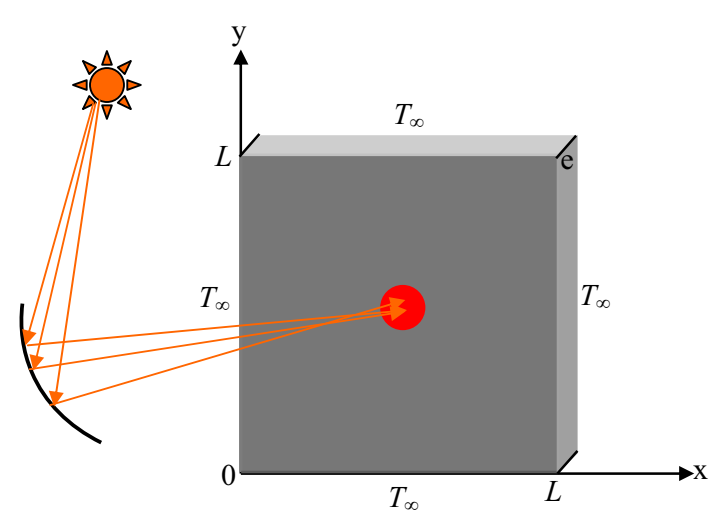

Fig. 7. Representative scheme of geometric boundary conditions at the receiver flat.

using the Poisson integral which takes into account the initial and boundary conditions, the final solution will be written in the following form:

$$
\begin{aligned}
T-T_{0}=\frac{\dot{Q}_{\text {in }}}{\rho_{r}\left(C_{p}\right)_{r}} & \frac{1}{8(\pi a t)^{3 / 2}} \\
& \times \exp \left[-\frac{\left(x-x_{0}\right)^{2}+\left(y-y_{0}\right)^{2}}{4 a t}\right]
\end{aligned}
$$

where $x_{0}$ and $y_{0}$ are the coordinates of the focal point which coincides with the sunspot surface center. The maximum temperature is reached at the point $P(x, y)$ which verifies the system of equations:

$$
\left\{\begin{array}{l}
\frac{\partial T}{\partial x}=0 \\
\frac{\partial T}{\partial y}=0
\end{array}\right.
$$

The system of equations (16) admits as solution $(x, y)=$ $\left(x_{0}, y_{0}\right)$. This result shows that the maximum temperature is produced in the center of the focal zone. The determination of the temperature field at the focal zone makes it possible to evaluate the heat flux distribution; this distribution is obtained analytically, through the determination of the absorbed heat flux per unit area of each mass element $\Delta m$ constituting the receiver flat.

Therefore, for a mass element $\rho \Delta V$, the absorptive heat flux is equivalent to the variation of the heat quantity received per time unit defined by:

$$
\dot{q}=\rho \Delta V\left(C_{p}\right)_{r} \frac{\mathrm{d} T}{\mathrm{~d} t}
$$

By deriving the temperature given in equation (15), the preceding expression becomes:

$$
\dot{q}=\rho \Delta V\left(C_{p}\right)_{r}\left(T-T_{0}\right)\left[\frac{\left(x-x_{0}\right)^{2}+\left(y-y_{0}\right)^{2}}{4 a t^{2}}-\frac{3}{2 t}\right]
$$

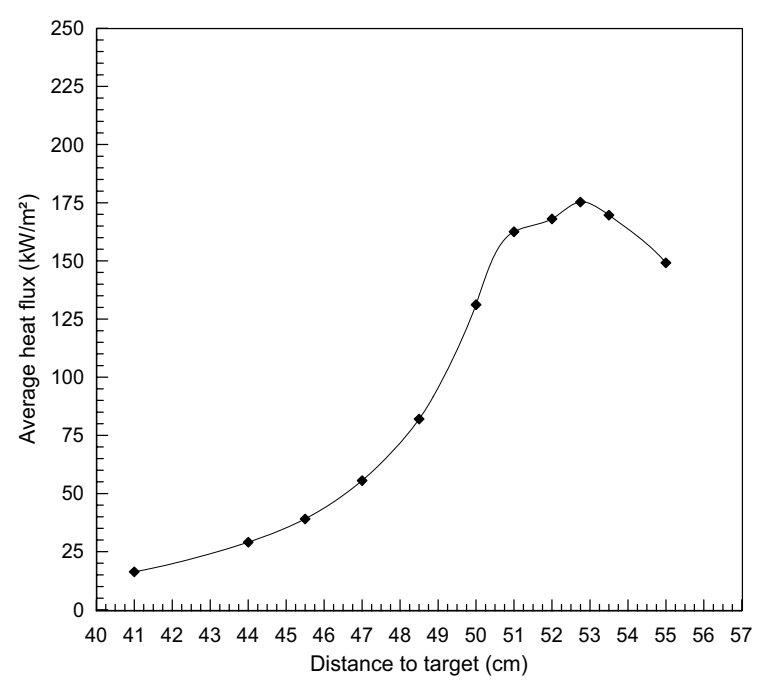

Fig. 8. Average heat flux at various positions of the receiver flat determined by Soltrace code.

Since $\Delta V=e \Delta x \Delta y$, the heat flux per area unit takes the following form:

$$
\dot{q}_{s}=e \rho\left(C_{p}\right)_{r}\left(T-T_{0}\right)\left[\frac{\left(x-x_{0}\right)^{2}+\left(y-y_{0}\right)^{2}}{4 a t^{2}}-\frac{3}{2 t}\right]
$$

A numerical simulation was performed to evaluate the temperature field as well as the heat flux distribution at the focal zone. This two dimensional numerical model based on the analytical development was developed using Matlab software. A geometric mesh was adopted by the program according to the geometry of the receiver flat, while the receiver thermophysical properties and geometric dimensions of the parabolic concentrator are introduced into a data file. The most important assumptions taken in this model are: the receiver flat is considered as an infinite solid, and their thermophysical properties (diffusivity, thermal conductivity, density) are independent of the temperature. As an application example, we take the mild steel receiver flat used in the experimental part and which is positioned in the center of the focal zone. Its dimensions are of $10 \times 10 \mathrm{~cm}$ and a thickness of $0.8 \mathrm{~mm}$, giving a ratio $\frac{e}{L}$ of 0.008 which verifies the condition of the infinite solid. A square grid $(\Delta x=\Delta y=1 \mathrm{~mm})$ was adopted in this program so that, the grid of the receiver flat counts 10000 nodes.

\section{Soltrace application}

\subsection{Flux heat distribution}

The Soltrace code based on Monte Carlo ray tracing method is a very powerful tool to solve the heat flux distribution of solar concentrator and receiver system. In this study, the Soltrace code was used in order to determine the heat flux concentrated on the receiver flat at various positions along the focal axis. The simulation parameters 
(a)

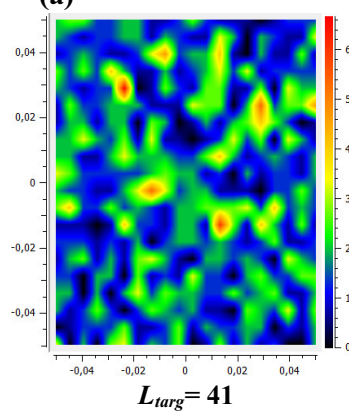

(d)

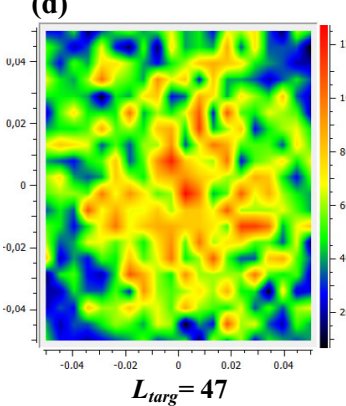

(g)

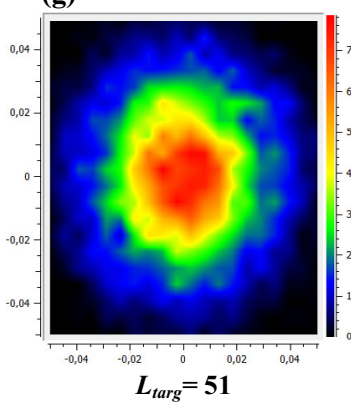

(b)

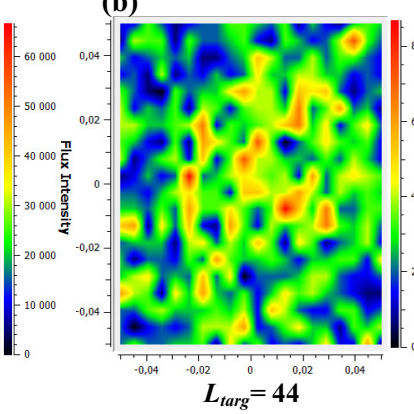

(e) (c)

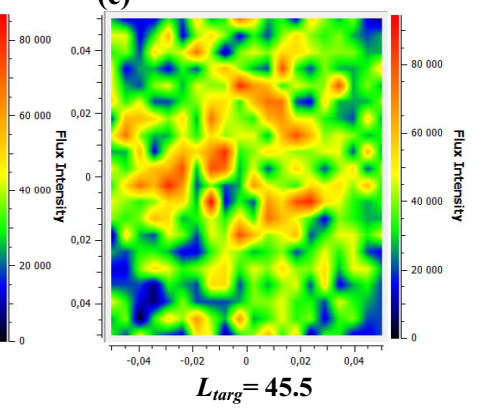

(f)

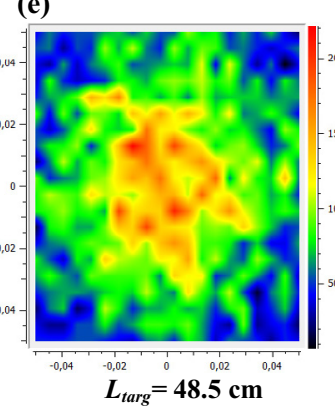

(h)

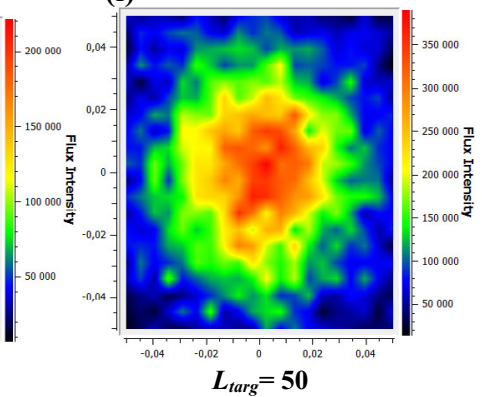

(i)
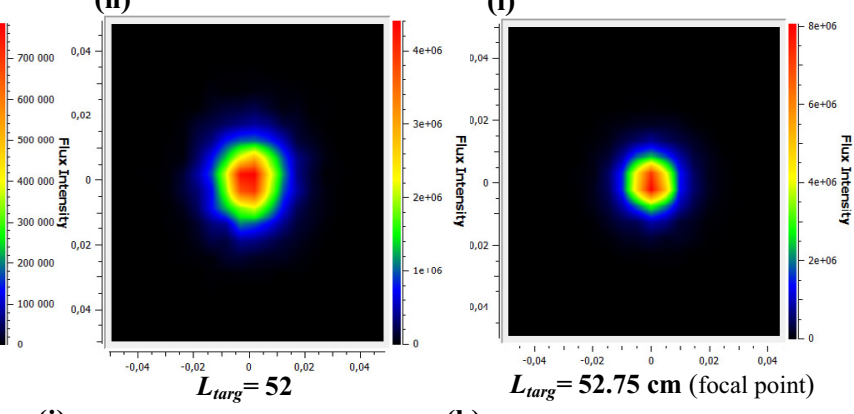

(k)
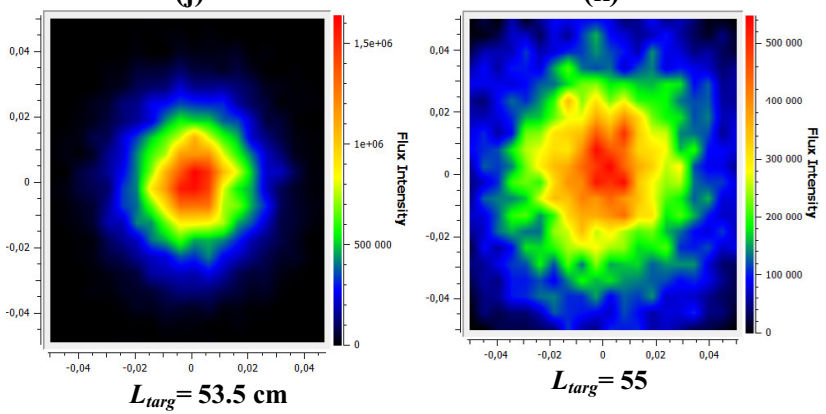

Fig. 9. Heat flux intensity $\left(\mathrm{W} \cdot \mathrm{m}^{-2}\right)$ calculated by the Soltrace code at various positions of the receiver flat.

introduced into the application take account all geometrical and optical characteristics of the concentrator and receiver flat used in experiment part, while the slope error is taken for $0.95 \mathrm{mrad}$ and the Gaussian error type is adopted. The average heat flux and the heat flux intensity at each position of the receiver flat are represented respectively in Figures 8 and 9.

\subsection{Receiver temperature}

The knowledge of the average heat flux makes it possible to determine the temperature of the receiver flat through the heat balance applied to the control volume delimiting its border, as indicated in the following expression:

$$
q_{\mathrm{avg}}=\underbrace{\varepsilon_{\mathrm{r}} \sigma T_{r}^{4}}_{\text {Radiated heat }}+\underbrace{\left(1-\varepsilon_{r}\right) q_{\mathrm{avg}}}_{\text {Absorbed heat }}+\underbrace{h\left(T_{\mathrm{r}}-T_{\infty}\right)}_{\text {Convective heat loss }}
$$

where $q_{\text {avg }}$ is the average heat flux focused on the receiver flat and $h$ is the average convective heat transfer coefficient expressed with the Nusselt number as follows:

$$
N u=\frac{h L}{K}
$$




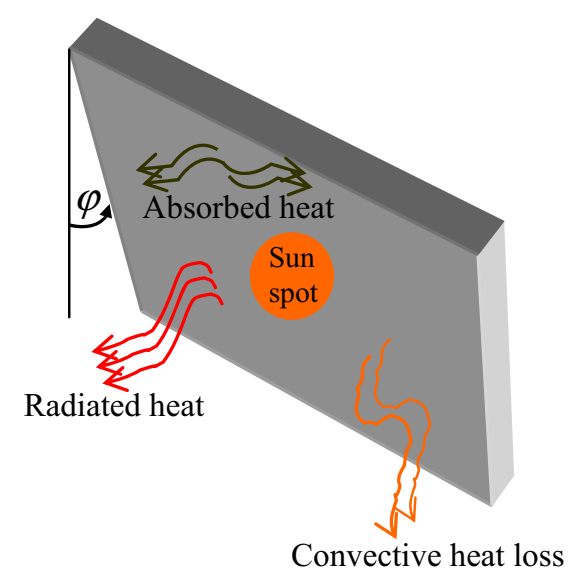

Fig. 10. Thermal balance scheme of the inclined receiver flat.

For the inclined plate downward facing under a constant heat flux (Fig. 10), Fujii and Imura [17] propose the following expression of Nusselt number in the range $10^{5}<\operatorname{GrPr} \cos \varphi<10^{11}$ :

$$
N u=0.56(G r \operatorname{Pr} \cos \varphi)^{\frac{1}{4}}
$$

where $\varphi$ is the inclined angle. The average Grashof number $G r$ and Prandt number $P r$ are defined by the following formulae respectively:

$$
\begin{aligned}
& G r=\frac{L^{3} g \beta\left(T_{\mathrm{r}}-T_{\infty}\right)}{v^{2}} \\
& \operatorname{Pr}=\frac{\mu\left(C_{\mathrm{p}}\right)_{\mathrm{air}}}{K}
\end{aligned}
$$

The volumetric thermal expansion coefficient $\beta$, the thermal conductivity $K$ and the thermal capacity of air are calculated by the following expressions $[7,18]$ :

$$
\begin{aligned}
\beta= & \frac{1}{T_{\infty}} \\
K= & 1.52 \times 10^{-11} T_{\infty}^{3}-4.86 \times 10^{-8} T_{\infty}^{2} \\
& +1.02 \times 10^{-4} T_{\infty}-3.93 \times 10^{-3} \\
\left(C_{\mathrm{p}}\right)_{\text {air }}= & 1.06 \times 10^{3}-0.499 T_{\infty}+1.14 \times 10^{-3} T_{\infty}^{2} \\
& -8 \times 10^{-7} T_{\infty}^{3}+1.93 \times 10^{-3} T_{\infty}^{4}
\end{aligned}
$$

If the expression of $h$ resulting from equation (21) is introduced into equation (20), the expression of this latter takes the following form:

$$
\varepsilon_{r} \sigma T_{\mathrm{r}}^{4}+0.56 K\left(\frac{g \beta}{L v^{2}} \operatorname{Pr} \cos \varphi\right)^{\frac{1}{4}}\left(T_{\mathrm{r}}-T_{\infty}\right)^{\frac{5}{4}}=0
$$

By the application of the iterative method, the preceding equation can be solved, which gives the temperature of the receiver flat under a given average heat flux at each position as indicated in Figure 11.

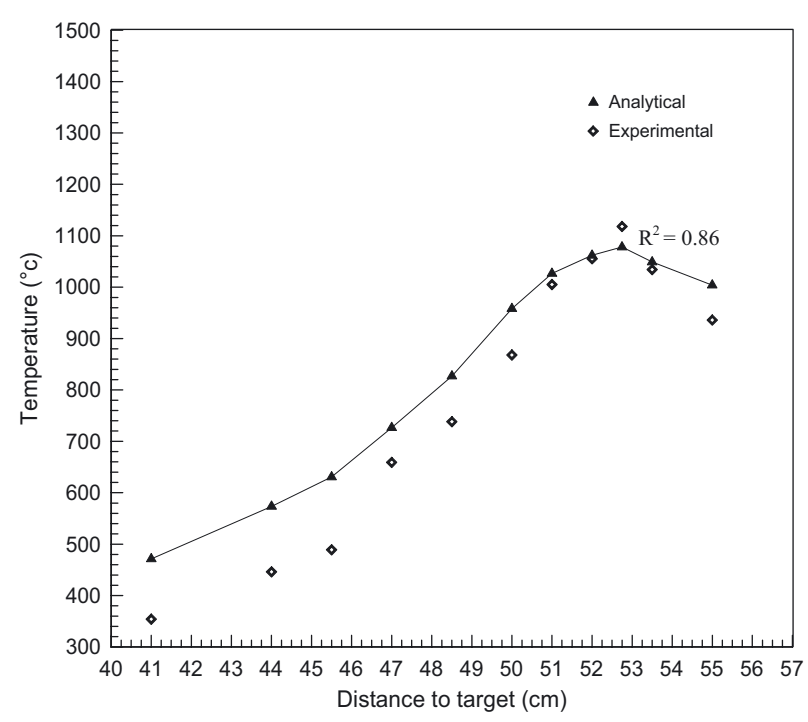

Fig. 11. Superposition of analytical and measured temperatures vs. target position.

\section{Result and discussion}

The results obtained through the numerical model are confronted with those obtained in experimental part, and those calculated by the application of the Soltrace code.

Figure 8 shows the variation of the average heat flux according to the distance from the receiver along the focal axis. The maximum average heat flux of $175 \mathrm{~kW} \cdot \mathrm{m}^{-2}$ is recorded at a distance to target equal to $52.75 \mathrm{~cm}$.

In Figure 9 the distributions of the heat flux intensity at each distance from the receiver are represented. It appears clearly that the flux intensity increases gradually to a peak of $8 \mathrm{MW} . \mathrm{m}^{-2}$ indicated in Figure 9i which coincides with the focal zone of the parabolic concentrator.

Figure 11 illustrates the measured temperatures in experiments and those obtained analytically according to each position of the receiver flat. The experimental results show that the temperature increases up to a maximum of $1118{ }^{\circ} \mathrm{C}$, recorded at a distance of $52.75 \mathrm{~cm}$ locating exactly the position of the focal zone that corresponds to the real focal distance from the solar concentrator. Once the real focal distance is deduced, we positioned the metal receiver flat which covered by a black matt painting exactly in the focal zone. After a short duration the sunspot was focused on metal flat by leaving a trace of gray colour, this spot is of circular form with a diameter equal to $4.1 \mathrm{~cm}$, equivalent to the surface of $13.19 \mathrm{~cm}^{2}$. The knowledge of the sunspot surface leads us to determine the real concentration ratio which takes a value of 1266 . The small difference of $1 \mathrm{~mm}$ between the theoretical focal distance given in Table 2, and that measured in experiments is unimportant. But it explains the good choice adopted in the positioning of the 152 facets which cover the reflecting surface of the parabolic concentrator, thing which reduces the slope error.

The standard error is about 0.86 meaning a good agreement between the results obtained by the analytical 


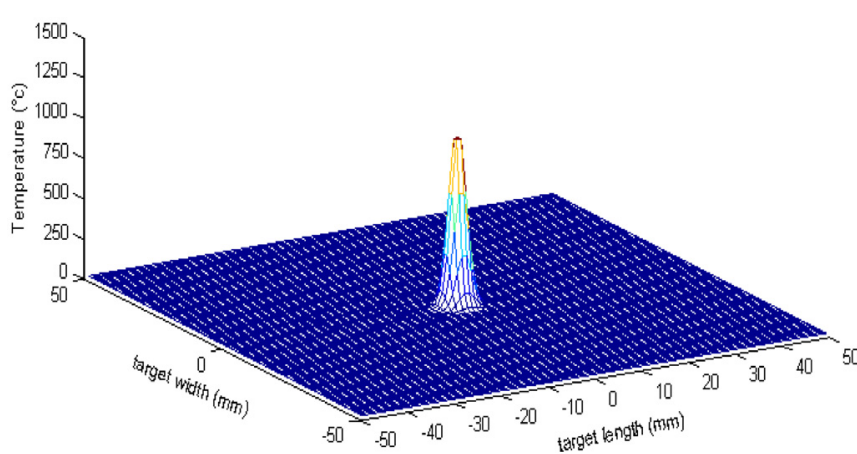

Fig. 12. Surface plot of the temperature distribution in receiver flat at the focal zone determined by the analytical model.

development and those given by experiments; this convergence becomes very appreciable in the distance range between $51 \mathrm{~cm}$ and $53 \mathrm{~cm}$ along the focal axis. Moreover we note that at the distance of $52.75 \mathrm{~cm}$ the temperature given by analytical development is maximum, which corresponds to the same found in experiments.

In Figure 12 is plotted the temperature distribution on the surface of the receiver flat at the focal zone determined by the analytical model. It is noted that the maximum temperature of $1075{ }^{\circ} \mathrm{C}$ is reached at the focal point under a direct normal insolation of $956 \mathrm{~W} \cdot \mathrm{m}^{-2}$. This peak of temperature is lower of $43^{\circ} \mathrm{C}$ than that found in experiments; this variation of temperature recorded between the experimental value and that provided by the analytical model can be explained by the fact that the analytical model takes a constant value of the thermal diffusivity of the receiver flat without taking account of its significant variation with the rise in temperature.

Figure 13 represents the distribution of solar flux focused on the receiver flat at the focal plan. The heat flux intensity records a maximum value of $11.38 \mathrm{MW} \cdot \mathrm{m}^{-2}$ at the focal point. This distribution of heat flux makes it possible to delimit the perimeter of the sunspot which takes a circular form with a diameter of $1.4 \mathrm{~cm}$ equivalent to the surface of $1.54 \mathrm{~cm}^{2}$.

Moreover the surface distribution of the solar flux in the focal zone gives the possibility to visualize two distinct zones; the first delimited by a diameter of $10 \mathrm{~mm}$ is characterized by strong heat flux intensity, while the second ranging between $10 \mathrm{~mm}$ and $14 \mathrm{~mm}$ is slightly affected by the intensity of flux relative to the first. The peak heat flux obtained by the application of the Soltrace code as indicated in Figure $9 \mathrm{i}$ is of $8.3 \mathrm{MW} \cdot \mathrm{m}^{-2}$ which represents a difference of $27 \%$ with the peak flux calculated by the analytical model.

\section{Conclusion}

The work presented in this paper is the development of an experimental and analytical methodology in order to characterize the geometrical, optical and thermical parameters of a solar parabolic concentrator. The suitable

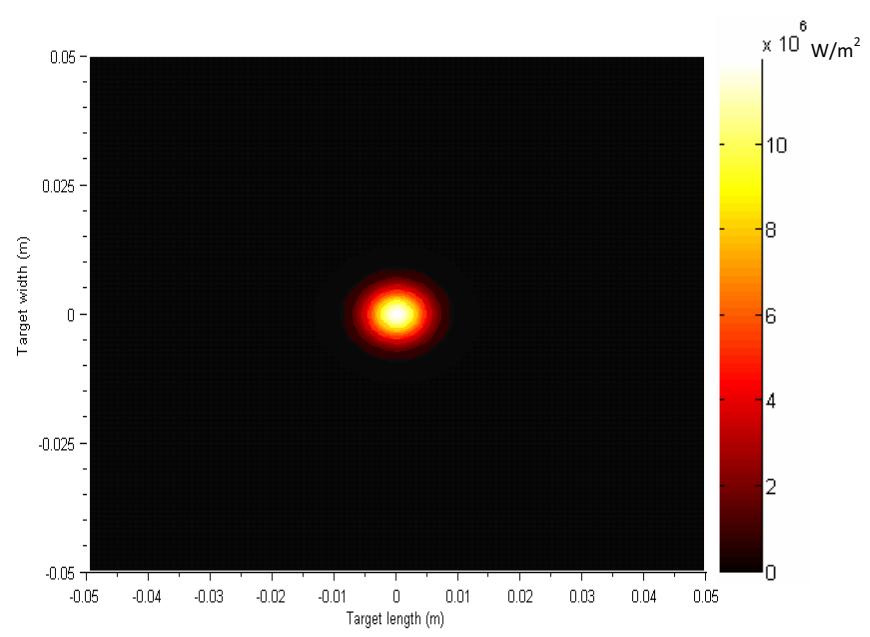

Fig. 13. Solar flux distribution at the focal zone determined by the analytical model.

choice of the dimensions of the facets of aluminium which constitute the reflective surface of the paraboloid as their adherence on primary surface played a significant role in the reduction of the slope error factor, and thus a better concentration ratio. This observation can be explained by the value of the theoretical focal length which is very close to that given in experiments. The experimental analysis shows that average flux at the focal zone is about 1.21 MW. $\mathrm{m}^{-2}$, while the power available in this zone is $1.64 \mathrm{KW}$ under a direct normal insolation of $956 \mathrm{~W} . \mathrm{m}^{-2}$. Moreover it should be noted that the determination of the focal distance by the analytical model remains in good adequacy with that calculated in experiments. The maximum temperature of $1118{ }^{\circ} \mathrm{C}$ reached at the focal zone requires that the materials with which the absorber is designed have to obtain a high melting point in order to avoid any deformation or damage which can be caused by a prolonged exploitation. This maximum temperature which is taken in experiments is in strong correlation with that obtained through the developed analytical model. The comparison between the peak heat flux that is obtained by the numerical model and that calculated by ray tracing technique is almost equal which shows an acceptable convergence of the analytical model.

\section{References}

[1] K. Lovegrove, G. Burgess, J. Pye, A new $500 \mathrm{~m}^{2}$ paraboloidal dish solar concentrator, Sol. Energy 85 (2011) 620-626

[2] S. Khanna, S.B. Kedare, S. Singh, Analytical expression for circumferential and axial distribution of absorbed flux on a bent absorber tube of solar parabolic trough concentrator, Sol. Energy 92 (2013) 26-40

[3] D. Riveros-Rosas, M. Sanchez-Gonzalez, C.A. ArancibiaBlunes, C.A. Estrada, Influence of the size of facets on point focus solar concentrators, Renewable Energy 36 (2011) 966-970 
[4] G. Zanganeh, R. Bader, A. Pedretti, M. Pedretti, A. Steinfeld, A solar dish concentrator based on ellipsoidal polyester membrane facets, Sol. Energy 86 (2012) 40-47

[5] G. Johnston, Focal region measurements of the $20 \mathrm{~m}^{2}$ tiled dish at the Australian National University, Sol. Energy 63 (1998) 117-124

[6] F.Q. Wang, Y. Shuai, Y. Yuan, H.P. Tan, C.M. Yu, Thermal performance analysis of porous media receiver with concentrated solar irradiation, Int. J. Heat Mass Transfer 62 (2013) 247-254

[7] F.Q. Wang, J. Tan, Y. Shuai, H.P. Tan, S. Chu, Thermal performance analyses of porous media solar receiver with different irradiative transfer models, Int. J. Heat Mass Transfer 78 (2014) 7-16

[8] F.Q. Wang, R. Lin, B. Liu, H.P. Tan, Y. Shuai, Optical efficiency analysis of cylindrical cavity receiver with bottom surface convex, Sol. Energy 90 (2013) 195-204

[9] R.W. Bliss, Notes on performance design of parabolic solar furnaces, Sol. Energy 1 (1957) 22-29

[10] A. Rabl, Comparison of solar concentrators, Sol. Energy 18 (1976) 93-111

[11] N. Hernandez, D. Riveros-Rosas, E. Vengas, R.J. Dorantes, A. Rojas-Moran, O.A. Jaramillo, C.A. Arancibia-Blunes, C.A. Estrada, Conical receiver for a paraboloidal concentrator with large rim angle, Sol. Energy 86 (2012) 1053-1062
[12] J.A. Harris, T.G. Lenz, Thermal performance of solar concentrator/cavity receiver systems, Sol. Energy 34 (1985) 135-142

[13] Shuang-Ying Wua, Lan Xiao, Yiding Cao, You-Rong Li, Convection heat loss from cavity receiver in parabolic dish solar thermal power system, Sol. Energy 84 (2010) 13421355

[14] F. Nepveu, A. Ferriere, F. Bataille, Thermal model of a dish/Stirling system, Sol. Energy 83 (2009) 81-89

[15] A. Steinfeld, M. Schubnell, Optimum aperture and operating temperature of a solar cavity receiver, Sol. Energy 50 (1993) 19-25

[16] M. Prakash, S.B. Kedare, J.K. Nayak, Determination of stagnation and convective zones in a solar cavity receiver, Int. J. Thermal Sci. 49 (2010) 680-691

[17] T. Fujii, H. Imura, Natural-convection heat transfer from a plate with arbitrary inclination, Int. J. Heat Mass Transfer 15 (1972) 755-767

[18] Z.Y. Wu, C. Caliot, F.W. Bai, G. Flamant, Z.F. Wang, Coupled radiation and flow modelling in ceramic foam volumetric solar air receivers, Sol. Energy 85 (2011) 23742385 